\title{
Interleukin-4 retards liver fibrosis in BALB/c mice model of carbon tetra-chloride (CCl4)-associated with decreased CD80 and interleukin-12 expressions
}

Johnny Amer ( $\sim$ j.amer@najah.edu )

An-najah National University Faculty of Medicine and Health Sciences

Suhaib Hattab

An-najah National University Faculty of Medicine and Health Sciences

Bilal Rahal

An-najah National University Faculty of Medicine and Health Sciences

Research article

Keywords: Liver injury, wound healing, macrophages, Vitamin D receptor

Posted Date: September 24th, 2019

DOl: https://doi.org/10.21203/rs.2.14914/v1

License: (c) (1) This work is licensed under a Creative Commons Attribution 4.0 International License.

Read Full License

Version of Record: A version of this preprint was published at Gastroenterology: Medicine \& Research on June 6th, 2019. See the published version at https://doi.org/10.31031/GMR.2019.03.000559. 


\section{Abstract}

Background: BALB/c mice showed easily induced Th2-type responses in several infection models. Certain macrophage phenotypes contribute to liver fibrosis. We characterized changes in macrophages phenotype (M1/M2) during fibrogenesis in liver fibrosis mice model.

Methods: Carbon-tetrachloride (CCl 4 ) hepatic-fibrosis was induced in BALB/c mice. Liver macrophages isolated were identified for M1 and M2 by CD80/iNOS and CD273 (programmed death ligand 2 (PDL2)/CD206, respectively. IL-4 were induced i.p along week-2 to week-4 of CCl 4 . Liver proteins were assessed for aSMA expressions, histology and ALT levels.

Results: CCl 4 -induced hepatic-fibrosis showed increased CD273 (from 20.1\% $\$ 3.1$ in naïve mice to $27.8 \%$ \pm 3.2 in fibrotic mice; $P=0.01$ ) while gradual decrease in CD80 (from $12 \% \pm 6.2$ in naïve mice to $1.97 \% \pm 0.4$ in fibrotic mice; $P<0.02$ ). The overall data showed decreased M1/M2 ratio. M2-macrophages showed inhibited expressions of IFN-g, IL-12 and vitamin-D-receptor (VDR) while high TGF-b levels. ALT, H\&E staining intensities and aSMA showed gradually increased along fibrosis while metabolic markers of serum insulin, vitamin D and VDR decreased. IL-4 inductions while inhibited fibrosis it elevated metabolic markers.

Conclusions: M2-macrophages express less IFN-g and IL-12, which might indicate inability differentiation of naive T cells into Th1 cells. IL-4 has an anti-fibrotic effects through antagonize M2-macrophages of TGF-b and ameliorating insulin, vitamin D, VDR and consequently attenuated liver-fibrosis.

\section{Background}

Chronic liver diseases can progress to cirrhosis, which is characterized by excessive accumulation of scar tissue (extracellular matrix, ECM) that is mainly composed of fibrillar collagens, glycoproteins and proteoglycans and that leads to severe distortion of the liver vascular architecture [1]. While activated myofibroblasts and hepatic stellate cells (HSCs) are the major producers of the fibrotic scar, their fibrogenic activation and proliferation depends on a complex interplay with other resident or recruited cells and their secreted factors [2]. Here, immune cells, which promote or attenuate fibrogenesis, have become targets of antifibrotic treatments [2,3,4]. Macrophages are central players of the immune response following tissue damage. Activated macrophages of different phenotypes are routinely classified into M1-macrophages and M2-macrophages. The classically activated M1-macrophages comprise immune effector cells with an acute inflammatory phenotype [5,6]. These are highly aggressive against bacteria and produce large amounts of lymphokines [7]. The alternatively activated, antiinflammatory M2-macrophages have various different functions, including regulation of immunity, maintenance of tolerance and tissue repair/ wound healing [8]. In additions, resident macrophages may originate from Ly-6C- CCR2- monocytes and have the potential to transform into classically (M1) or alternatively (M2) polarized macrophages in vivo [9]. M1- and M2-type polarizations can be induced in vitro by cytokines that also favor T helper (Th) 1 vs Th2 cell polarization, i.e., by IFNy and IL-12 vs IL- 4 
and IL-13, respectively [10]. M1-type macrophages are considered pro-inflammatory and potentially antifibrotic, while the role of M2-type macrophages, which can be subdivided into at least 5 subtypes, in inflammation and fibrosis is less well defined [11]. The various M2-subtypes may exhibit either pro- or anti-fibrotic activity $[12,13]$. This and other observations suggest that the overall functional relevance of the classical M1 and M2 paradigm is limited [14]. The purpose of this study was to study liver macrophages and effects of interleukin-4 in an experimental model of carbon tetrachloride $\left(\mathrm{CCl}_{4}\right)$ mediated liver injury in BALB mice.

\section{Methods}

Animals

Male mice on the $B A L B / c$ background, 12 weeks of age, weighing $22 \pm 0.5 \mathrm{~g}$, received care according to ethic regulations of the An-Najah National University and NIH guidelines. Mice were purchased commercially from Harlan Laboratories, Jerusalem-Israel. All animal protocols were approved by the institutional animal care ethical committee at the An-Najah National University, and housed in a barrier facility.

\section{Fibrosis-model}

Carbon-tetrachloride ( $\mathrm{CCl}_{4}$; Sigma, C-5331) fibrosis-model was induced by intraperitoneal (I.P) injections at $0.5 \mu \mathrm{l}$ pure $\mathrm{CCl}_{4} / \mathrm{g}$ body weight (1:9 dilution in mineral oil) biweekly, from 1 to 4 weeks. The time of sacrifice was performed 2 days after the final $\mathrm{CCl}_{4}$ injections. The animals were terminated $72 \mathrm{hr}$ after the final $\mathrm{CCl}_{4}$ injection intramuscularly with $0.1 \mathrm{ml}$ of ketamine: xylazine: acepromazine (4:1:1) per $30 \mathrm{~g}$ of body-weight.

\section{Histological assessmentsof liver injury}

The posterior one third of the liver was fixed in $10 \%$ formalin for 24 hours and then paraffin-embedded in an automated tissue processor. Seven-millimeter liver sections were cut from each animal. Sections (15 $\mathrm{mm}$ ) were then stained in $0.1 \%$ sirius red F3B in saturated picric acid (both from Sigma). Hematoxylin and eosin (H\&E) staining was performed for each animal. Knodell score was assessed blindly by an expert hepatic pathologist (M. I. F.) based on H\&E and Sirius red staining, using the modified Histological Activity Index (HAl) criteria, incorporating semi quantitative assessment of periportal/periseptal interface hepatitis ( $0-4)$, confluent necrosis $(0-6)$, focal lytic necrosis/apoptosis and focal inflammation (0-4), portal inflammation (0-4), and architectural changes/fibrosis and cirrhosis (0-6). F4/80 stain was applied in liver sections to allocate macrophage infiltrations.

\section{IL-4 treatment}


Mouse recombinant IL -4 was purchased from R\&D systems (USA). Multiple IL-4 injections were performed by injecting the mice twice a week with intraperitoneal (i.p.) injections of IL $-4(0.2 \mu \mathrm{g} / 0.5 \mathrm{ml}$ phosphate-buffered saline (PBS)/mouse) with from week 0 to week $2 \mathrm{CCl}_{4}$ inductions and from week 2 to week 4 of $\mathrm{CCl}_{4}$ inductions. Sham treatment was performed by i.p. injections of PBS.

Serum alanine aminotransferase (ALT)

Blood samples were collected from the inferior vena cava and ALT was measured using an automated enzymatic assay with the Vistros Chemistry Systems 950.

\section{Serum ELISA measurements}

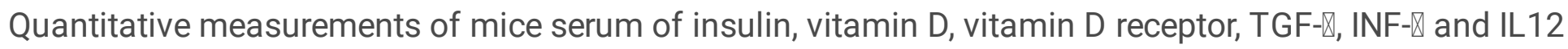
(all purchased from abcam; USA) were determined according to the manufacture instructions.

\section{Macrophage isolations}

Macrophages were isolated by perfusion of the liver with digestion buffer. After perfusion, the liver was homogenized and incubated at $37^{\circ} \mathrm{C}$ for $30 \mathrm{~min}$. The digested liver cell suspension was centrifuged to remove hepatocytes and cell clumps. The supernatant was then centrifuged to obtain a pellet of cells depleted of hepatocytes to a final volume of $1 \mathrm{ml}$. Macrophages were then isolated from this cell suspension using $24 \%$ metrizamide gradient separation [15].

\section{Tissue RNA extraction}

Total cellular RNA was extracted from target-frozen tissues using Trizol reagent ${ }^{\circledR}$. Synthesized $\beta$-actin and SMA were detected by Real-Time PCR: $\beta$-actin (as a housekeeping-gene) - Forward: 5'-GAT-GAG-ATTGGC-ATG-GCT-TT-3', ß-actin -Reverse: 5'-AGA-GAA-GTG-GGG-TGG-CTT-TT-3'. aSMA -Forward: 5'-TCCTCC-CTG-GAG-AAG-AGC-TAC-3', aSMA - Reverse: 5'-TAT-AGG-TGG-TTT-CGT-GGA-TGC-3'.

\section{Alpha smooth muscle actin immunoblot}

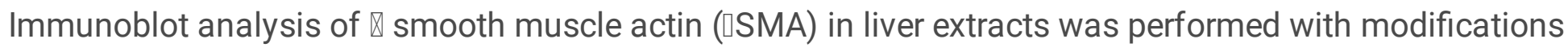
as previously described [16].

\section{Flow cytometry}

To determine macrophages purity, cells were fixed with $4 \%$ paraformaldehyde for 10 minutes and permeabilized with $0.1 \%$ saponine in PBS for 20 minutes, then stained with anti-CD 45 (FITC); anti-CD 16+ (Pacific blue) and anti-CD68 (APC) (IQ Products, Groningen) for $30 \mathrm{~min}$ at room temperature. Additional antibodies to further immune-phenotype macrophages were used; anti-CD80 (PE) (R\&D systems USA), anti-CD273 (PerCP) (R\&D systems USA), iNOS (Alexa-Fluor 488) and anti-CD206 (FITC) both from Thermofisher Scientific, Invitrogen USA were used. For macrophages phenotype alterations; Rat AntiMouse IFN-y (PE), Mouse TGF-beta RII Antibody (PE) and Mouse IL-12 R beta 1 Antibody (PE), all from 
(R\&D systems USA). For Vitamin D receptor, rabbit anti-VDR unconjugated-antibody was used (abcam, USA); as a secondary antibody, anti-rabbit-APC was used (R\&D systems USA). All stained cells were analyzed with a flow cytometer (FACS-calibur, Becton-Dickinson, Immunofluorometry systems, Mountain View, CA).

\section{Viability assay}

\section{The viability of macrophages was determined by trypan blue staining. In briefly, $100 \mu$ l of cells was aseptically} transferred to a $1.5 \mathrm{ml}$ clear tube and incubated for $3 \mathrm{~min}$ at room temperature $\left(25^{\circ} \mathrm{C}\right)$ with an equal volume of $0.4 \%$ (w/v) trypan blue solution (Sigma, USA). Cells were counted using a dual-chamber hemocytometer and a light microscope. Nonviable cells were stained blue and viable cells were unstained. These two types of cells were recorded separately, and the means of six independent cell counts were pooled for analysis.

Statistical analysis

Statistical differences were analyzed either with the 2-tailed unpaired Student's t-test (For comparison between two groups) or one-way analysis of variance (one-way ANOVA with Newman-Keuls' post-tests among multiple groups) using Graph pad Prism 5.0 (GraphPad software, La Jolla, CA). Data are shown as means $\pm \mathrm{SEM}$.

\section{Results}

Elevated frequencies of programmed death ligand 2 (PDL2) in macrophages of BALB/c mice model of liver fibrosis associated with decreased CD80 counts

Polarizing of monocytes into M1 and M2 macrophages are an important step in liver fibrosis progression and regression [17]. We characterized their role during fibrogenesis. Hepatic fibrosis was induced in $\mathrm{BALB} / \mathrm{c}$ mice by biweekly IP $\mathrm{CCl}_{4}$ injections for 4 weeks and was compared with naive vehicle-treated mice.Liver macrophages were isolated as described in materials and methods along week 1 to week 4 of $\mathrm{CCl}_{4}$ injections. Macrophages were then stained for $\mathrm{M} 1$ and $\mathrm{M} 2$ subpopulations for $\mathrm{CD} 80$ and antiCD273, respectively. iNOS for M1 macrophages and anti-CD206 for M2 macrophages were also used as an additional marker to profile macrophages subpopulations. Fig. 1A shows a represented dot plot of 
flow cytometry analysis of the isolated polymorphonulcear cells (Gate 1; PMN following Ficoll separations according to their size (FSC-H) and granularity (SSC-H). Fig. 1B shows the whole population stained with the pan-leukocytes marker anti-CD45-FITC. A gate (Gate 2) was drawn on our potential macrophages populations identified as CD45-positive cells with high SSC-A. For further identifying our macrophages, Fig. 1C demonstrates plotted population stained with both anti-CD68-APC (panmacrophages marker) and a gate (Gate 3) was set on CD68-poistive population. We also confirmed panmacrophages populations with staining with F480-pacrific blue and similar results were obtained (data not shown) Fig. 1D shows a representative distribution of macrophages subpopulations of M1 and M2 identified as CD80-PE and CD273-PerCP, respectively. Of the total evaluated CD45 ${ }^{+} / C D 68+$ cells, averages of around $13.3 \%$ of CD80 and $12.4 \%$ of iNOS M1-macrophages were expressed in naïve mice. These percentages were decreased in early fibrosis ( $\mathrm{w} 1$ and $\mathrm{w} 2$ of $\mathrm{CCl}_{4}$ ) and ended in advanced fibrosis of w4 to $1.97 \%$ and $5.1 \%$, respectively (Fig. $1 \mathrm{E} ; \mathrm{P}<0.05$ ). In contrast, expressions of CD273 and CD206 on M2-macrophages of naïve mice were $19.8 \%$ and $35.6 \%$, respectively. Both CD273 and CD206 expressions showed to be elevated significantly through fibrosis severities to $27.9 \%$ and $48 \%$, respectively $(p<0.05)$. There were no significance differences in CD273 and CD206 expressions between week 1 along week 4 of fibrosis (Fig. 1F).The overall M1 to M2 ratio showed a decreased in M1/M2 percentages (Fig. 1G), indicating an alterations in macrophages subpopulations counts where M2 were the dominant populations and increased in fibrosis while M1 showed decreased in their counts.

M2-macrophages phenotype alterations accompanied by decrease in IL-12, VDR expressions and

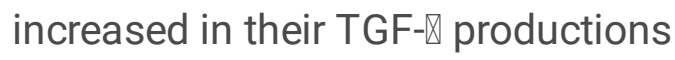

In order to evaluate whether changes in macrophages count were accompanied with phenotypic

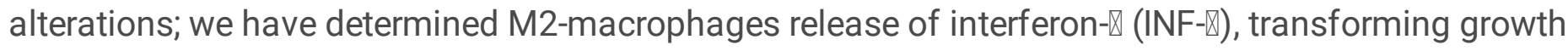

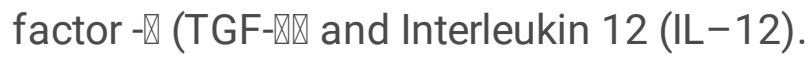

$\mathrm{IL}-12$ is produced by activated antigen-presenting cells (dendritic cells, macrophages) [18]. It promotes the development of Th1 responses and is a powerful inducer of IFNY production by T and NK cells [19]. TGFQX is a powerful modulator of inflammation, and has been implicated in the regulation of leukocyte and vascular endothelial cell activation [20]. Mice lacking the TGF咽 gene die of widespread inflammatory disease either in utero or shortly after birth [21, 22].

Fig. 2A shows flow cytometry analysis of the percentages of INF- $₫$ significantly decreased from $65.4 \pm 11 \%$ in naïve mice to $55.4 \pm 9 \%(w 1, p=0.04), 51.9 \pm 7 \%(w 2, p=0.03), 45.8 \pm 5 \%(w 3, p=0.03)$, and $36.5 \pm 5 \%$ $(w 4, p=0.001)$. In parallel, Fig. 2B shows IL12 percentages were also gradually decreased from $13.4 \pm 3.9 \%$ in naïve mice to $10.375 \pm 2.7 \%$ ( $w 1, p=0.04), 4.025 \pm 1.6 \%(w 2, p=0.03), 3.7 \pm 0.3 \%(w 3, p=$ 0.03 ), and $1.075 \pm 0.1 \%(w 4, p=0.001)$. In contrast to these data, TGF- $\square$ expressions were gradually elevated in the M2-macrophages from $19.5 \pm 3.7 \%$ in naïve mice to $29.0 \pm 5.1 \%, 35.1 \pm 7.9 \%, 41.3 \pm 7 \%$, and $51.5 \pm 9 \%$ in $w 1, w 2, w 3$ and $w 4$, respectively (Fig. 2C, $p<0.05$ between all groups). M2-macrophages expressions of VDR were $55.9 \pm 8.1 \%$ in naïve mice and were gradually decreased to $49 \pm 8 \%, 40 \pm 6 \%$, $37 \pm 5.8 \%$ and $29 \pm 4.1 \%$ in $w 1, w 2, w 3$ and $w 4$, respectively (Fig. 2D, $p<0.05$ between all groups). These 
results suggest M2-macrophages lost their inflammatory response exhibiting INF- $\$ during fibrosis progressions (reduce INF- - ), probably due to decrease in their IL-12 secretions, and in contrast they had an anti-inflammatory response through their release to TGF-ndDecrease in VDR expression on M2macrophages during fibrosis progressions could suggest its correlation with their pro-fibrogenic properties

Interleukin-4 ameliorated metabolic markers of insulin, vitamin D and vitamin D receptor associated with $\mathrm{CCl}_{4}$ fibrosis model

We sought to further explore changes in phenotype of the dominant M2-macrophages populations; therefore, we specified metabolic markers for their correlation to macrophages activity through inductions of interleukin-4 (IL-4) in an acute and chronic model of $\mathrm{CCl}_{4}$. IL-4 showed to restore insulin sensitivity [23] and in other studies speeds up fibrosis reversal [24]. In our current study, we evaluated serum insulin levels, vitamin $D$ as well as vitamin $D$ receptor. Results from preclinical studies showed that vitamin $D$ (or calcitriol) administration reduced the levels of blood glucose and improved insulin sensitivity in diabetic mice [25] and attenuated the fibrosis [26].

Fig. 3 shows effects of IL -4 on serum insulin, vitamin $D$ and vitamin $D$ receptor levels in acute injections of $\mathrm{CCl}_{4}$ (2 weeks) as well in chronic injections of $\mathrm{CCl}_{4}$ (4 weeks). Fig. 3A shows no significant changes in serum insulin levels in the acute phase of the disease as compared to mice with no $\mathrm{CCl}_{4}$ treatments while the chronic phase of liver fibrosis exhibit low insulin levels. While inductions of i.p injections of IL -4 did not affect insulin levels in acute phase, IL-4 inhibited decrease in insulin levels in the chronic model of $\mathrm{CCl}_{4}(\mathrm{P}=0.01)$ and maintained serum insulin comparable to untreated mice. Similar data were obtained for serum vitamin D levels illustrated in Fig. 3B. In Fig. 3C, serum VDR showed to decrease in acute $\mathrm{CCl}_{4}$ injections from 730.35 in untreated groups to 376.25 in acute $\mathrm{CCl}_{4}$ injection with a further decrease to $282 \mathrm{ng} / \mathrm{ml}$ in chronic injections. IL-4 inductions while inhibited decrease in VDR in acute model it elevated serum VDR in chronic model to levels exceeding untreated group $(P=0.0012)$. IL-4 inductions in $\mathrm{CCl}_{4}$-untreated mice had similar patterns to naïve animals in all metabolic profile (data are not shown). The results emphasize the association between fibrosis progressions and alteration in metabolic profile such as insulin resistance. IL -4 is suggested to activate insulin receptor and promote signaling pathway

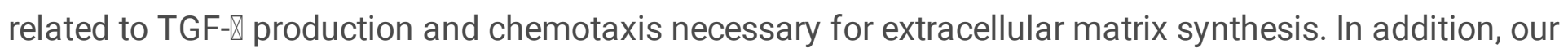
data refer to VDR as an early metabolic marker to predict fibrosis progressions or regressions.

Fibrotic BALB/c mice develop severe fibrosis and inflammation with increased intra-hepatic macrophages while reversed by $I L-4$

In order to evaluate whether our metabolic data together with the effects of IL-4 are correlated with alteration in fibrosis progressions; we have performed fibrosis marker of the aSMA expressions, ALT levels and histological liver assessments. 
H\&E immunohistochemical staining (Fig. 4A) for the animal groups showed lymphocytes infiltration in the fibrotic mice that were attenuated with IL -4 inductions. No inflammatory infiltrates were seen in $\mathrm{H} \& \mathrm{E}$ staining of naïve untreated. Fig. 4B shows the lack of fibrosis stained by Sirius-Red in naïve untreated mice. $\mathrm{CCl}_{4}$ induction showed increased formation of red fibrosis septae in $\mathrm{CCl}_{4}$ and less prominent in IL4 induce fibrotic mice. To define infiltrate of macrophages in these livers; F4/80 stain for the macrophage marker was assessed. Fig.4C shows cell infiltrate including macrophages (F4/80-positive cells) in the fibrotic animals. Fibrotic mice with IL-4 showed similar staining to untreated mice with less macrophage infiltrates.

Fig. 4D shows expressions of aSMA-mRNA in livers with and without fibrosis with IL-4 inductions. $\mathrm{CCl}_{4^{-}}$ induction provoked increased fibrosis and aSMA expression was significantly increased $4.2 \pm 0.5$ folds in the $\mathrm{CCl}_{4}$ animals as compared to the untreated counterparts $(P=0.018)$. Fibrotic animals with $\mathrm{IL}-4$ had reduced expressions of aSMA mice as compared to animals with the $\mathrm{CCl}_{4}$ alone $(P=0.03)$ and had similar expressions to untreated mice $(P=n s)$. These results confirmed same pattern obtained from histology assessments. There were no differences in the CYP2e activity of liver extracts from two fibrotic groups (data not shown). Serum ALT levels (Fig. 4E) were significantly $(P<0.05)$ increased from $57.3 \pm 18.7$ in naïve mice to $228 \pm 29,404.5 \pm 89,333.3 \pm 77$ and $178 \pm 51 \mathrm{IU} / \mathrm{L}$ in $\mathrm{CCl}_{4}$ - fibrotic mice of week $1,2,3$ and 4 , respectively. On the other hand, animals with 2 weeks of $\mathrm{CCl}_{4}$ were induced with $\mathrm{IL}-4$ had serum ALT levels reduced following 1 week of induction with prominent reductions in ALT levels following 2 weeks ( $p$ $=0.01)$. The ALT results were in line with obtained H\&E staining.

Serum obtained from fibrotic mice following IL -4 inductions showed increased levels of TGF- $\triangle$ (Fig. 4 F; $P$

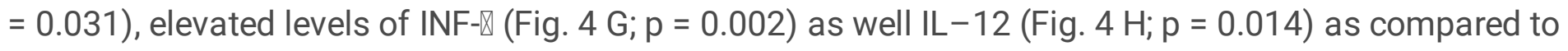
fibrotic mice with no inductions. These results indicate IL -4 ability to accelerate liver fibrosis (through TGF- $₫$ ) and therefore inhibit hepatic stellate cells activations (decreased in 口SMA), minimize liver injury through differentiation of macrophages by IL -12 and promote INF- $\$ expressions and as a consequent enhance their activity. Taking all together, $\mathrm{IL}-4$ showed to enhance liver inflammation and fibrosis progression thorough ameliorating vitamin D, VDR and insulin suggesting IL -4 effects as speeds up fibrosis reversal.

\section{Discussion}

Macrophage polarization is a process whereby macrophages phenotypically mount a specific functional response to the microenvironment [27]. In the host immune system, macrophages have an important role in both normal and disease conditions. Macrophages mainly sustain homeostasis by mediating the release of pro-inflammatory and anti-inflammatory cytokines [28]. In this study we have adapted the chemical model of liver fibrosis; $\mathrm{CCl}_{4}$; in $\mathrm{BALB} / \mathrm{c}$ mice in order to study macrophages interference during fibrosis progressions. The mechanisms of acute $\mathrm{CCl}_{4}$ hepatotoxicity involve immediate cleavage of $\mathrm{CCl}_{4}$ by cytochrome P450 2E1 (CYP2E1) in hepatocytes [29], which generates trichloromethyl radical, leading to lipid peroxidation and membrane damage [30]. Subsequently, activated hepatic macrophages (Kupffer 
cells) produce toxic mediators (e.g., inflammatory cytokines, reactive oxygen intermediates, eicosanoids), resulting in the injury of parenchymal cells [31]. In contrast to C57BL/6 mice, BALB/c mice produce high levels of IL -4 [32]. IL -4 together with IL-13 and their common receptor subunit IL-4Ra play a central role in switch for M1- towards M2-type macrophage polarization and Th2 cell polarization which are held responsible for the promotion of organ fibrosis [33, 34].

We have showed that during fibrosis progression, M2-macrophages release of IFN- $\gamma$ and IL-12 were decreased. These two cytokines known to contribute to the polarization of T cells to a T helper type 1 (Th1) phenotype and may also induce the differentiation of monocytes to macrophages while simultaneously triggering the production of chemokines attracting monocytes and granulocytes, such as CXC chemokine ligands CXCL8, CXCL9 and CXCL10, [35] as well as stimulating the phosphorylation of the signal transducer and activator of transcription 4 (STAT-4) protein [27]. Notably, we showed that macrophages frequencies are altered during liver fibrosis progression; gradual decrease in CD80 expressions (M1-macrophage subpopulation) were seen along fibrosis progressions while CD273 (M2macrophage subpopulation) were elevated. The overall data showed a decrease in $\mathrm{M} 1 / \mathrm{M} 2$ macrophage ratio in BALB/c mice model of liver fibrosis indicating a reduced differentiation of M1-macrophages.

In addition, we demonstrate that effects of IL -4 on macrophages promotes both hepatic inflammation and fibrosis regressions while it ameliorated levels of insulin, vitamin $D$ and vitamin $D$ receptor following $\mathrm{CCl}_{4}$ liver injury. There is an increasing evidence that vitamin D deficiency is associated with metabolic syndrome [36] and specific vitamin D receptor gene polymorphisms have been found to be related to the different components of metabolic syndrome [37]. Experiments done in high fat diet rats showed vitamin D supplementation ameliorated some of the abnormalities associated with metabolic syndrome, namely hyperinsulinemia, insulin resistance, hypertension and obesity as well as dyslipidemia when given alone. This was associated with evident attenuation of the hepatic, renal and cardiac structural abnormalities [26]. On the other hand, evidence for the central role of macrophages in insulin resistance is provided by recent studies demonstrating that deletion of PPARY in high fat diet mice prevents polarization of monocyte/macrophages to the M2 phenotype and impairs insulin action [38].

Our BALB/c fibrotic model might suggest inhibit differentiation of lymphocytes and $T$ cells through a

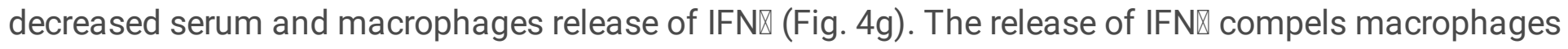
to secrete proinflammatory cytokines; leading them to generate toxic mediators in great quantities [39]. Lymphocytes activate macrophages by releasing either IFNX. On the other hand, increased serum and macrophages release of TGF- $\beta 1$ (Fig. 4F) could be a regulatory feedback mechanism to facilitate the resolution of the pro-inflammatory response. TGF- $\beta 1$ also triggers fibroblast activation and development of ECM-producing myofibroblasts that facilitate repair and drive fibrosis. In our data, IL-4 inductions further elevated TGF- $\beta 1$ serum levels. Furthermore, IL- 4 inductions ameliorate IL-12 levels in our mice model and could suggest differentiation of naive T cells into Th1 cells. It is known as a T cell-stimulating factor, which can stimulate the growth and function of T cells. It stimulates the production of INF-Y and TNF-a from T cells and natural killer (NK) cells [40]. We showed IL-4 mediated suppression of ALT levels and fibrosis histology of H\&E and Sirius red stain together with less inflammatory macrophages. In 
conclusion, besides $\mathrm{CCl}_{4}$ role in hepatotoxicity [41], it showed to interfere with the metabolic effects of

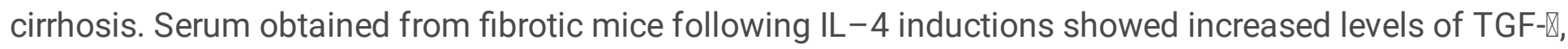
elevated levels of INF- $\forall$ and IL-12 as compared to fibrotic mice with no inductions. Our results indicate IL-4 ability to accelerate liver fibrosis (through TGF- - ) and therefore inhibit hepatic stellate cells activations (decreased in $\triangle S M A$ ), minimize liver injury through differentiation of macrophages by IL-12 and promote INF- $\square$ expressions and as a consequent enhance their activity. Taking all together, IL-4 showed to enhance liver inflammation and fibrosis progression thorough ameliorating vitamin $D, V D R$ and insulin suggesting IL-4 effects as speeds up fibrosis reversal. Macrophages phenotype modulations could be used as therapeutic target for liver fibrosis regressions through interventions with IL -4 .

\section{Conclusion}

The findings presented in the current study investigated association between alterations in macrophages phenotype sub-populations and progression of liver fibrosis. In addition, we observed changes in metabolic markers associated with chronic injections of $\mathrm{CCl}_{4}$ in our BALB/c mice model. An inverse correlation between serum insulin levels, vitamin $D$ and Vitamin $D$ receptor and stage of liver fibrosis were seen. Moreover, we found increased in liver M2-macrophages count during fibrosis and was associated with their reduced expressions of INF- $\mathbb{Z}, \mathrm{IL}-12$ and vitamin $\mathrm{D}$ receptor, while elevated expressions of TGF囚.

Increased serum and macrophages release of TGF- $\beta 1$ following IL- 4 inductions could be a regulatory feedback mechanism to facilitate the resolution of the pro-inflammatory response. TGF- $\beta 1$ also triggers fibroblast activation and development of ECM-producing myofibroblasts that facilitate repair and drive fibrosis. Furthermore, IL-4 inductions ameliorate IL-2 levels in our mice model and could suggest differentiation of naive T cells into Th1 cells.

Macrophages mainly sustain homeostasis by mediating the release of pro-inflammatory and antiinflammatory cytokines. M2-Macrophages of PDL2 alterations through IL-4 suggest macrophages as a therapeutic target for liver fibrosis regressions. Therefore, this study uncovers additional side of the effects of IL-4 in liver fibrosis associated with metabolic syndrome and emphasizes its role in modulating macrophages towards regression of fibrosis.

\section{Abbreviations}

VDR: Vitamin D receptor; HSCs: Hepatic stellate cell; $\mathrm{CCl}_{4}$ : Carbon tetrachloride; Vitamin D: 25-(OH) D3.

\section{Declarations}

Acknowledgment

Not Applicable 
JA designed, performed the experiments and wrote the manuscript; $\mathrm{SH}$ and BR revised the manuscript and analyzed the data.

\section{Funding}

An-Najah National University funded this work including materials disposables and submission fees under the grant number of ANNUN1718So004.

\section{Availability of data and materials}

All data are presented within the manuscript.

\section{Ethics approval}

The institutional animal care ethical committee of An-Najah National University approved all animal experiments and procedures.

\section{Consent for publication}

The authors indicated no potential conflicts of interest.

Competing interests

The authors indicated no potential conflicts of interest.

\section{Author details}

Dept. of Medical Sciences, Faculty of Medicine, An Najah National University, Nablus-Palestine.

\section{References}

1. Schuppan D, Afdhal NH. Liver cirrhosis. Lancet 2008; 371(9615): 838-51. 2. Puche JE, Saiman Y, Friedman SL. Hepatic stellate cells and liver fibrosis. Compr Physiol 2013; 3(4):1473-92. Review 3. Schuppan D1, Kim YO. Evolving therapies for liver fibrosis. J Clin Invest 2013; 123(5):1887-901. 4. Trautwein C, Friedman SL, Schuppan D, et al. Hepatic fibrosis: Concept to treatment. J Hepatology 2015; 62(1 Suppl):S15-24. Review 5. Liaskou E, Zimmermann HW, Li KK, et al. Monocyte subsets in human liver disease show distinct phenotypic and functional characteristics. Hepatology 2013, 57:385-98. 6. Tacke F, Zimmermann HW. Macrophage heterogeneity in liver injury and fibrosis. J Hepatology 2014; 60:10906. 7. Murray PJ, Wynn T. Obstacles and opportunities for understanding macrophage polarization. J Leukoc Biol 2011; 89(4): 557-63. 8. Shapouri-Moghaddam A, Mohammadian S, Vazini H, Taghadosi M, Esmaeili SA, et al. Macrophage plasticity, polarization, and function in health and disease. J Cell Physiol 2018; 233(9):6425-6440. Review. 9. Egawa M, Mukai K, Yoshikawa S, et al. Inflammatory monocytes recruited to allergic skin acquire an anti-inflammatory M2 phenotype via basophil-derived interleukin-4. 
Immunity 2013; 38(3):570-80. 10. Jaguin M, Houlbert N, Fardel O, et al. Polarization profiles of human MCSF-generated macrophages and comparison of M1-markers in classically activated macrophages from GM-CSF and M-CSF origin. Cell Immunol 2013; 281(1):51-61. 11. Gundra UM, Girgis NM, Gonzalez MA, et al. Vitamin A mediates conversion of monocyte-derived macrophages into tissue-resident macrophages during alternative activation. Nat Immunol 2017; 18(6):642-653. 12. Borthwick LA, Barron L, Hart KM, et al. Macrophages are critical to the maintenance of IL-13-dependent lung inflammation and fibrosis. Mucosal Immunol 2016; 9(1):38-55. 13. Sica A, Invernizzi P, Mantovani A. Macrophage plasticity and polarization in liver homeostasis and pathology. Hepatology 2014; 59(5):2034-42. 14. Murray PJ, Allen JE, Biswas SK, et al. Macrophage activation and polarization: nomenclature and experimental guidelines. Immunity 2014; 41(1):14-20. 15. Pei-zhi Li, Jin-zheng Li, Min Li, et al. An efficient method to isolate and culture mouse Kupffer cells. Immunology Letters. 2014; 158: 52-56. 16. Muhanna N, Abu-Tair L, Doron S, et al. Amelioration of hepatic fibrosis by NK cell activation. Gut 2011; 60(1):90-98. 17. Duffield JS, Forbes $\mathrm{SJ}$, Constandinou CM, et al. Selective depletion of macrophages reveals distinct, opposing roles during liver injury and repair. J Clin Invest 2005; 115:56-65. 18. Zheng H, Ban Y, Wei F, et al. Regulation of Interleukin-12 Production in Antigen-Presenting Cells. Adv Exp Med Biol 2016; 941:117-138. 19. Hsieh CS, Macatonia SE, Tripp CS, et al. Development of TH1 CD4+ T cells through IL-12 produced by Listeriainduced macrophages. Science 1993; 260 (5107): 547-9. 20. Letterio JJ, Roberts AB. Regulation of immune responses by TGF- $\bigotimes$. Annu Rev Immunol 1998; 16:137-61. 21. Shull MM, Ormsby I, Kier AB, et al. Targeted disruption of the mouse transforming growth factor- $₫ 1$ gene results in multifocal inflammatory disease. Nature 1992; 359:693-9. 22. Kulkarni AB, Huh CG, Becker D, et al. Transforming growth factor $\otimes 1$ null mutation in mice causes excessive inflammatory response and early death. Proc Natl Acad Sci USA 1993; 90:770-4. 23. Stafeev IS, Michurina SS, Podkuychenko NV, et al. Interleukin-4 Restores Insulin Sensitivity in Lipid-Induced Insulin-Resistant Adipocytes. Biochemistry 2018; 83(5):498-506. 24. Weng SY, Wang X, Vijayan S, Tang Y, et al. IL-4 Receptor Alpha Signaling through Macrophages Differentially Regulates Liver Fibrosis Progression and Reversal. E Bio Medicine 2018; 29:92-103. 25. Meerza D,

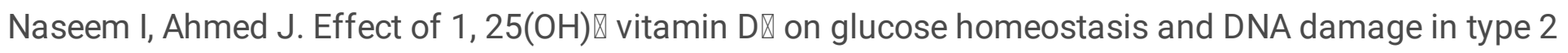
diabetic mice. J Diabetes Complications 2012, 26(5):363-8. 26. Mostafa DK, Nasra RA, Zahran N, et al. Pleiotropic protective effects of Vitamin $D$ against high fat diet-induced metabolic syndrome in rats: One for all. Eur J Pharmacol 2016; 792:38-47. 27. Wang N, Liang H, Zen K. Molecular mechanisms that influence the macrophage m1-m2 polarization balance. Front Immunol 2014; 5:614. 28. Das A, Sinha M, Datta S, et al. Monocyte and macrophage plasticity in tissue repair and regeneration. Am J Pathol 2015; 185:2596-2606. 29. Johansson I, Ingelman-Sundberg M. Carbon tetrachloride-induced lipid peroxidation dependent on an ethanolinducible form of rabbit liver microsomal cytochrome P-450. FEBS Lett 1985; 183: 265-269. 30. Recknagel O, Glende A, Dolak A, et al. Mechanisms of carbon tetrachloride toxicity. Pharmacol Ther 1989; 43:139-154. 31. Edwards J, Keller J, Kauffman C, et al. The involvement of Kupffer cells in carbon tetrachloride toxicity. Toxicol Appl Pharmacol 1993;119: 275-279. 32. Falcone M1, Rajan AJ, Bloom BR, et al. A critical role for IL-4 in regulating disease severity in experimental allergic encephalomyelitis as demonstrated in IL-4-deficient C57BL/6 mice and BALB/c mice. J Immunol 1998; 160(10):4822-30. 33. Cheever AW, Williams ME, Wynn TA, et al. Anti-IL-4 treatment of Schistosoma mansoni-infected mice inhibits development of $T$ cells and non-B, non-T cells expressing Th2 cytokines

Page 12/18 
while decreasing egg-induced hepatic fibrosis. J Immunol 1994; 153(2):753-9. 34. Chiaramonte MG, Schopf LR, Neben TY, et al. IL-13 is a key regulatory cytokine for Th2 cell-mediated pulmonary granuloma formation and IgE responses induced by Schistosoma mansoni eggs. J Immunol1999; 162(2):920-30. 35. Lech M, Anders HJ. Macrophages and fibrosis: How resident and infiltrating mononuclear phagocytes orchestrate all phases of tissue injury and repair. Biochim Biophys Acta 2013; 1832:989-997. 36. Gulseth HL, Gjelstad IM, Birkeland KI, et al. Vitamin D and the metabolic syndrome. Curr Vasc Pharmacol 2013; 11(6): 968-84. Review 37. Schuch NJ, Garcia VC, Vívolo SR, et al. Relationship between Vitamin D Receptor gene polymorphisms and the components of metabolic syndrome. Nutr J 2013, 2:96. 38. Charo I. Macrophage Polarization and Insulin Resistance: PPARy in Control. Cell metabolism 2007; 6 (2): 96-98. 39. Hasegawa Y, Inagaki T, Sawada M, Suzumura A. Impaired cytokine production by peripheral blood mononuclear cells and monocytes/macrophages in Parkinson's disease. Acta Neurol Scand 2000; 101:159-164. 40. Luckheeram R, Zhou R, Verma A, et al. CD4+T Cells: Differentiation and Functions. J of Immun Res 2012; 2012:1-12. 41. Mion F1, Géloën A, Agosto E, et al. Carbon tetrachloride-induced cirrhosis in rats: influence of the acute effects of the toxin on glucose metabolism. Hepatology 1996; 3(3):582-8.

\section{Figures}


Figure 1
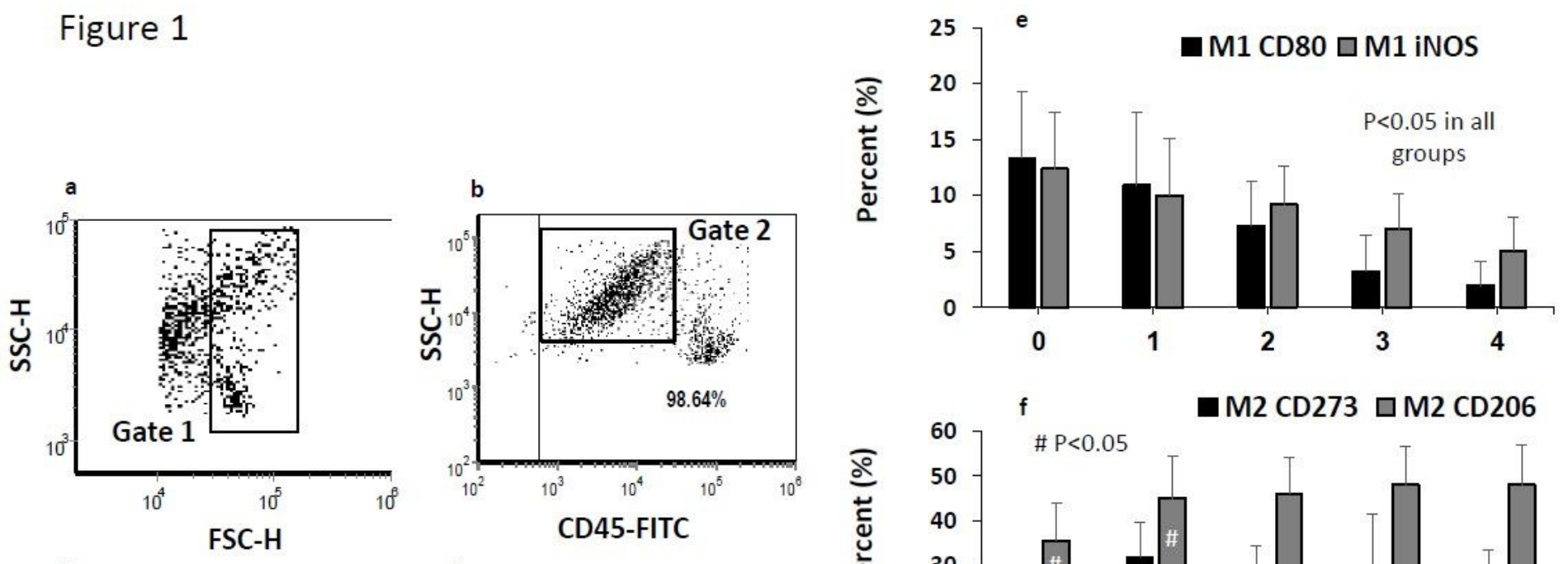

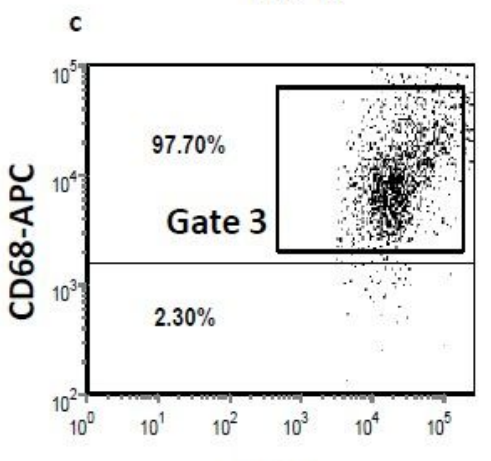

FSC-H

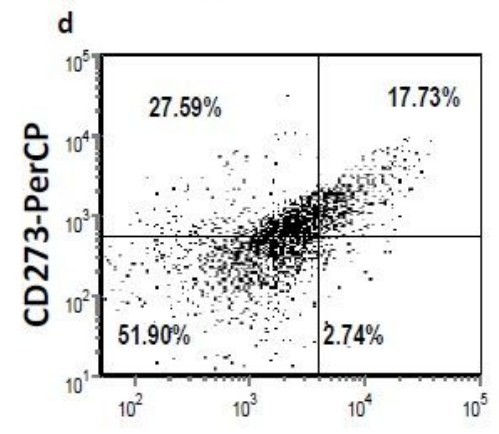

CD80-PE
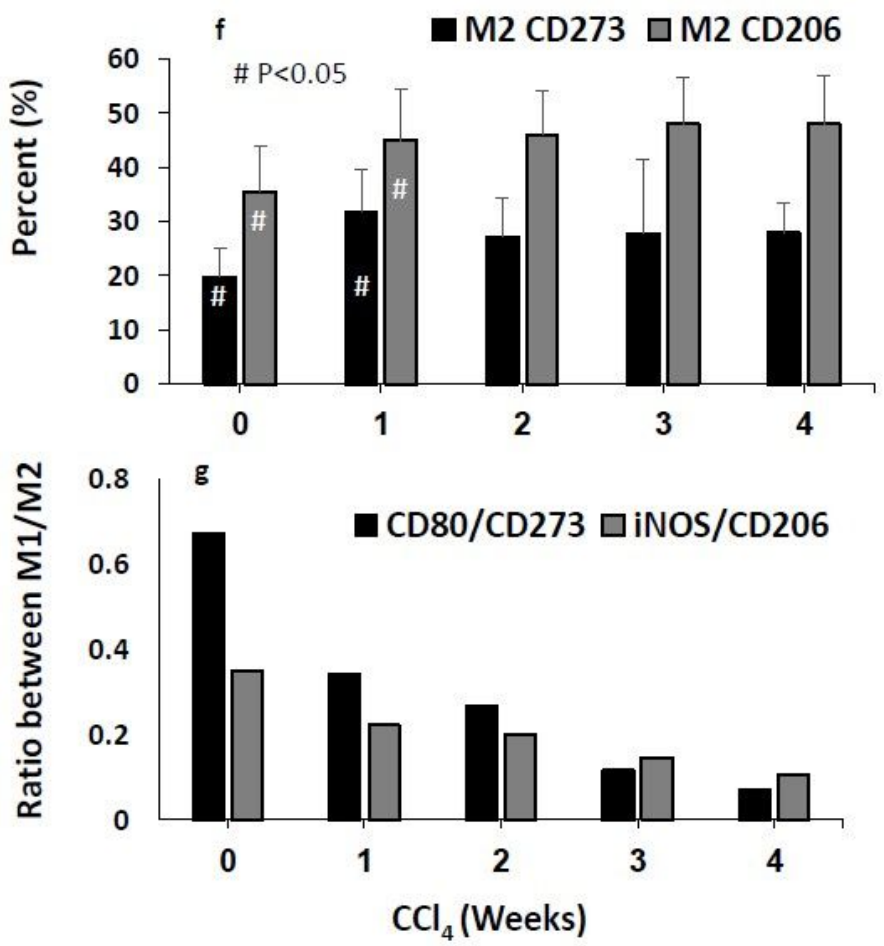

Figure 1

Frequency of macrophages subpopulations in mice fibrosis model. Flow cytometry was used to assess macrophages sub-populations $\mathrm{CCl} 4$ induce fibrosis of BALB/c mice. Liver macrophages were purified using the Ficoll-Paque method, as described in Materials \& Methods. a. Dot plot of cells by size (FSC-H) and granularity (SSC-H). Gate 1 was set on the PMN cells to exclude any remaining RBC and hepatocytes. b. Cells were stained for pan-leukocyte markers (CD45-FITC; 98.64\%) and the potential macrophages populations were gated (Gate 2) for CD45+ and high SSC-H. c. Cells from Gate 2 were further assessed for macrophages content by staining for CD68-APC (pan-mouse macrophages marker; $97.70 \%$ ); F4/80 was also used to confirm pan-macrophages population (data not shown). Macrophages CD68+ were gated (Gate 3). d. Macrophages sub-populations were stained for CD80-PE and iNOS-Alexa-Fluor 488 to identify the M1 populations and for CD273-PerCP and CD206-FITC to identify the M2 populations. Averages of M1-macrophages (e), M2-Macrophages ( $f$ ) (Results are expressed by mean $\pm S D$ ), and ratio of $\mathrm{M} 1 / \mathrm{M} 2$ macrophages subpopulations $(\mathrm{g})$ are plotted along week 1 to week 4 of liver fibrosis progressions. 
Figure 2
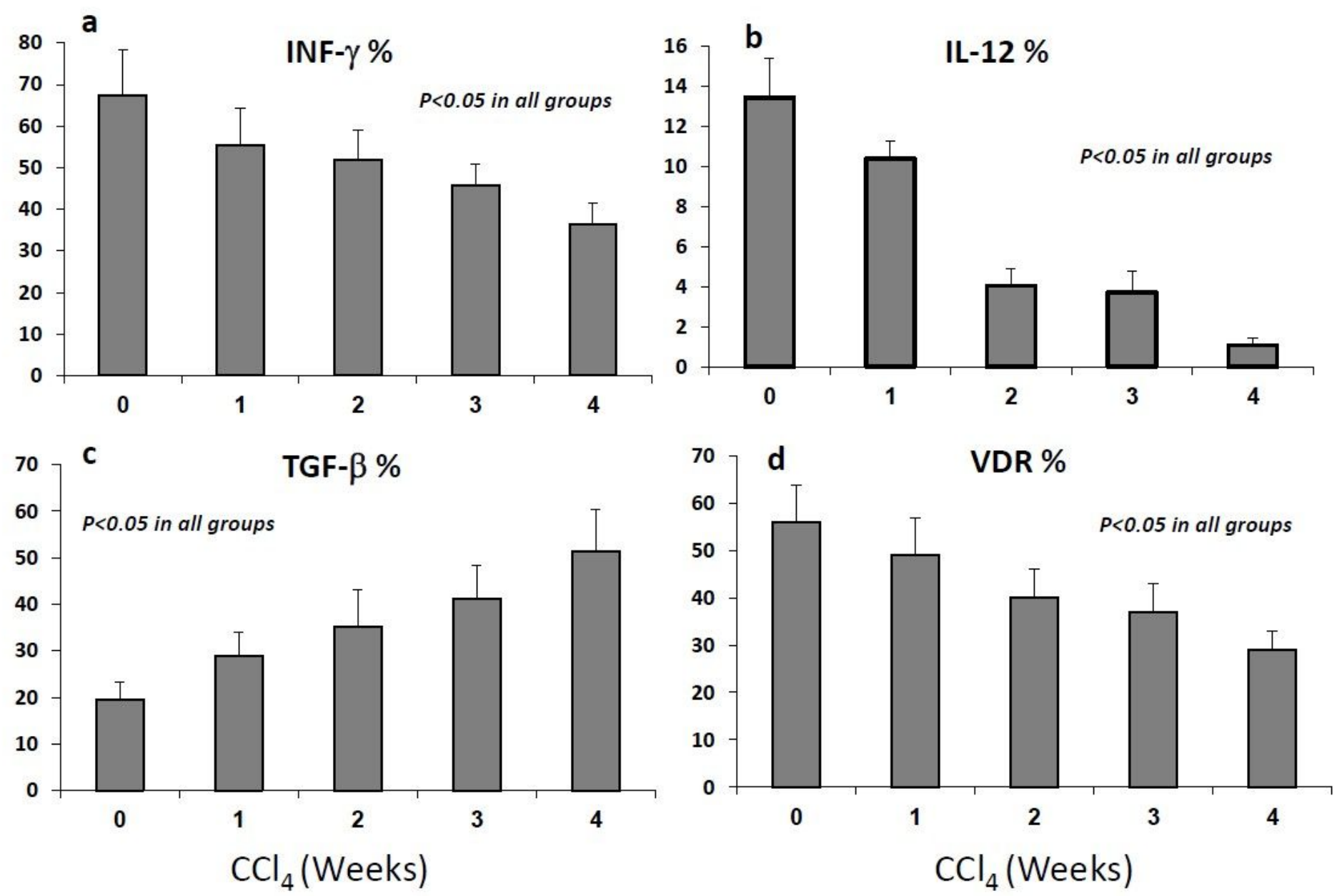

Figure 2

Changes in macrophages cytokine productions and VDR are correlated with fibrosis progressions. a. Percentages of INF- $囚$ expressions by M2-macrophages significantly decreased in early fibrosis stages (w1, w2), and decreased more with progressing fibrosis (w3-w4) $(P<0.05)$. b. Percentages of IL-12 expressions of macrophages decreased across fibrosis progressions $(P<0.05)$. c. Percentages of TGF- $\square$ expressions in M2-macrophages significantly increased along all fibrosis $(P<0.05)$. d. Percentages of VDR expressions in M2-macrophages significantly decreased along all fibrosis $(P<0.05)$. 
Figure 3
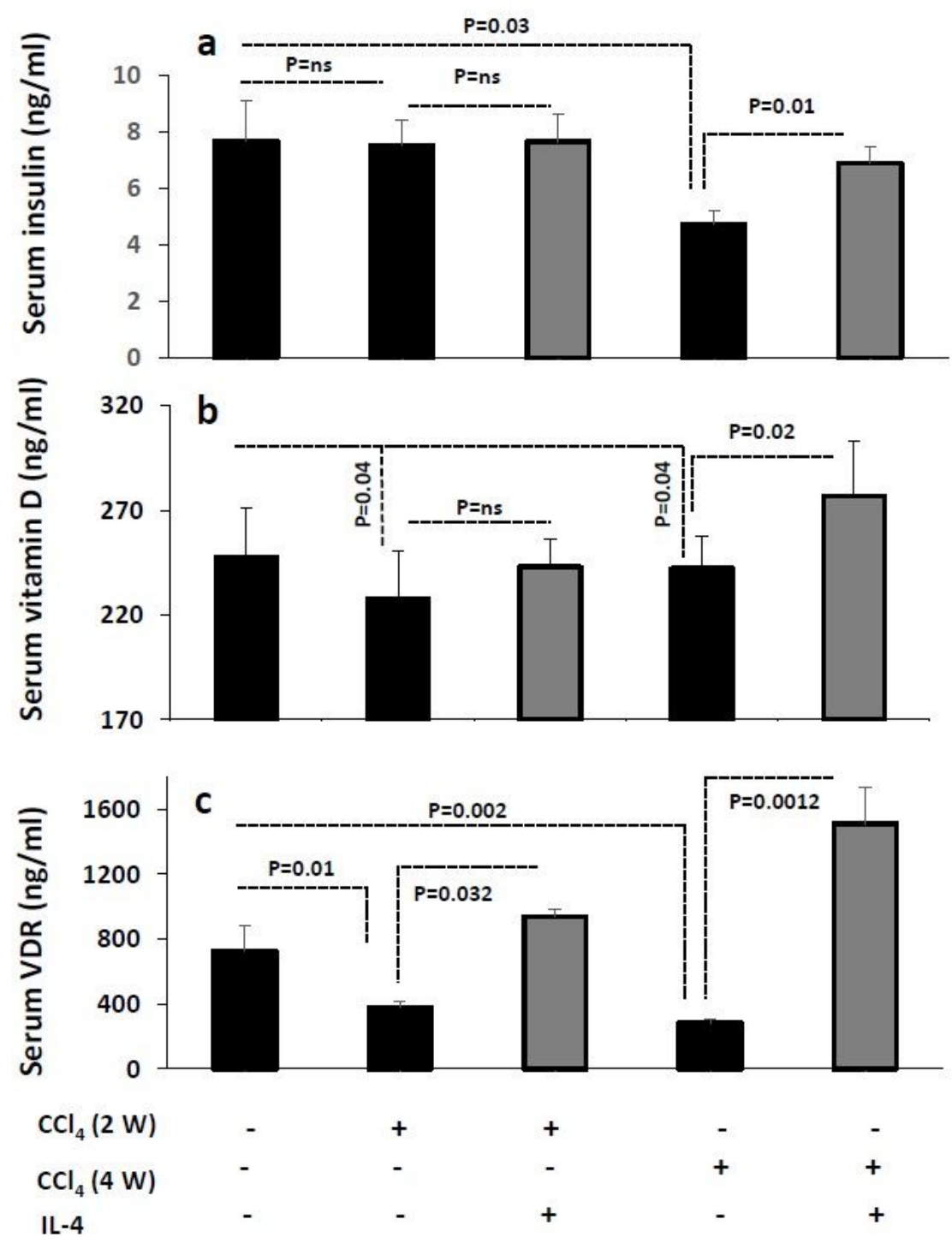

Figure 3

Interleukin-4 ameliorated metabolic makers associated with the $\mathrm{CCl} 4$ hepatotoxicity effects. Inductions of IL-4 in week 0 to week 2 following CCl4 had no significant difference on serum insulin levels ( $P=n s)(a)$ as well on serum vitamin D levels (b), while IL-4 induced form week 2 of CCl4 to week 4 elevated serum insulin levels $(a ; p=0.01)$ and vitamin $D(b ; p=0.02)$ following their significant reduction in week 4 fibrosis $(\mathrm{P}<0.05)$. C. VDR showed to be reduce following 2 weeks of $\mathrm{CCl} 4$ and a more prominent decrease was obtained following 4 weeks of CCl4. IL-4 have elevated VDR in both the acute the chronic injections of $\mathrm{CCl} 4(\mathrm{P}<0.03)$. 
Figure 4
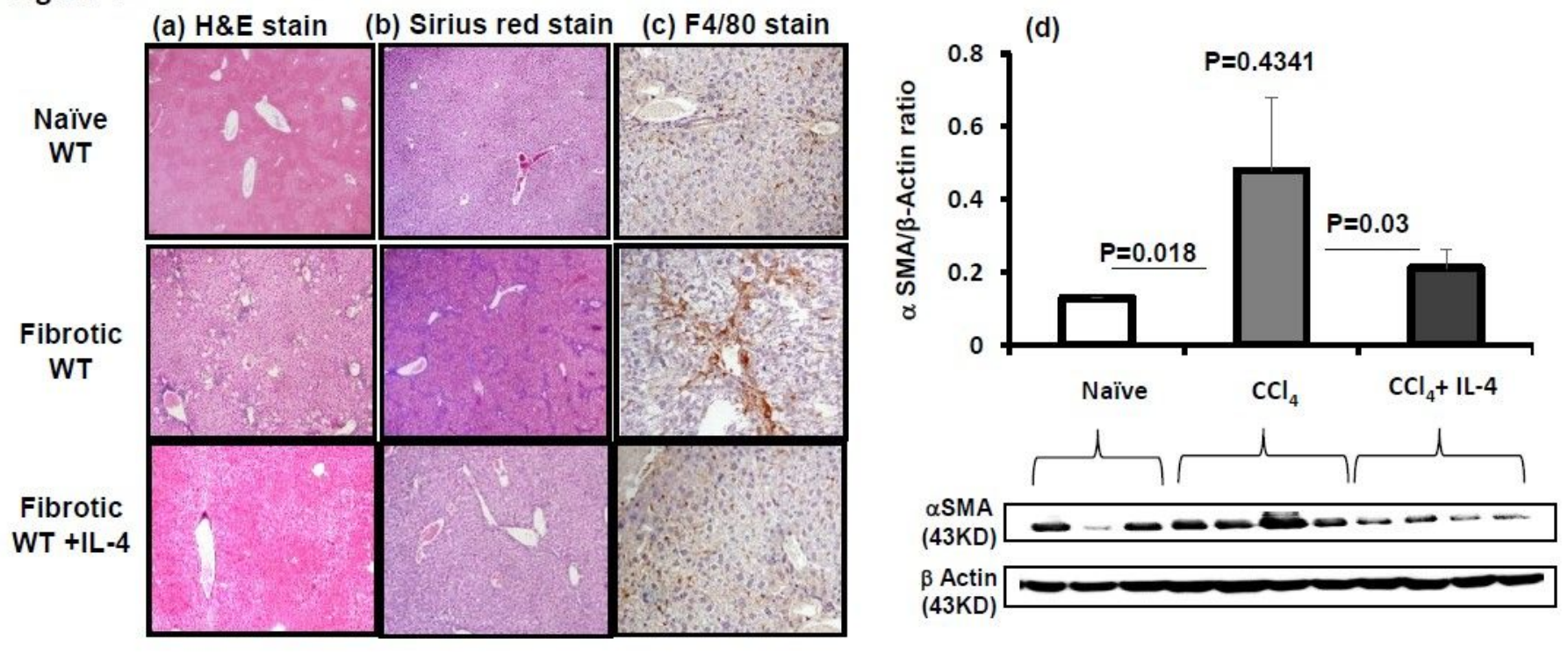

(e)
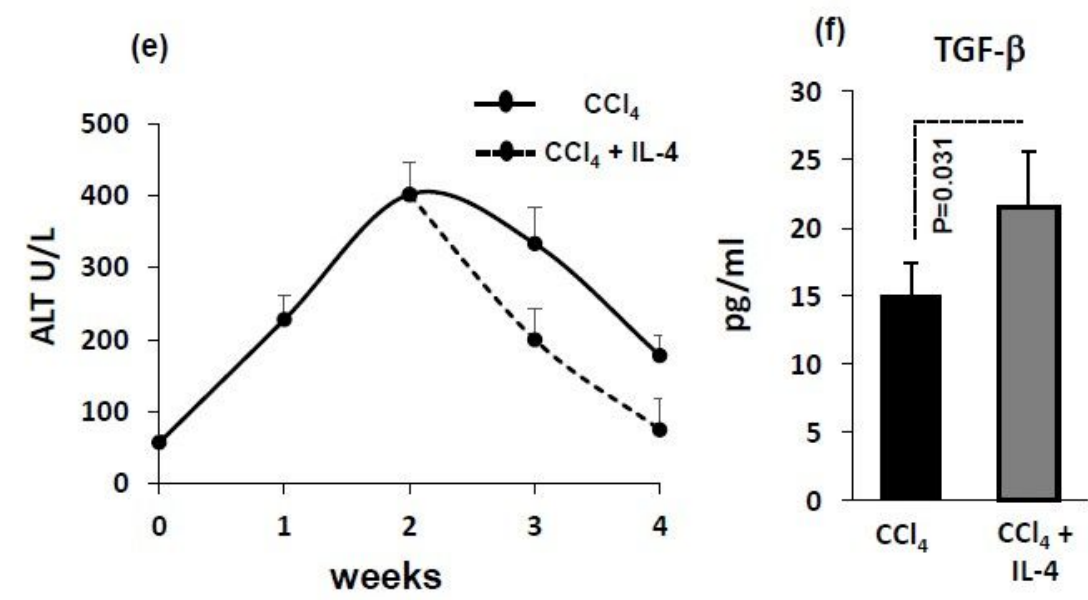

(g) INF- $\gamma$

(h) IL-12
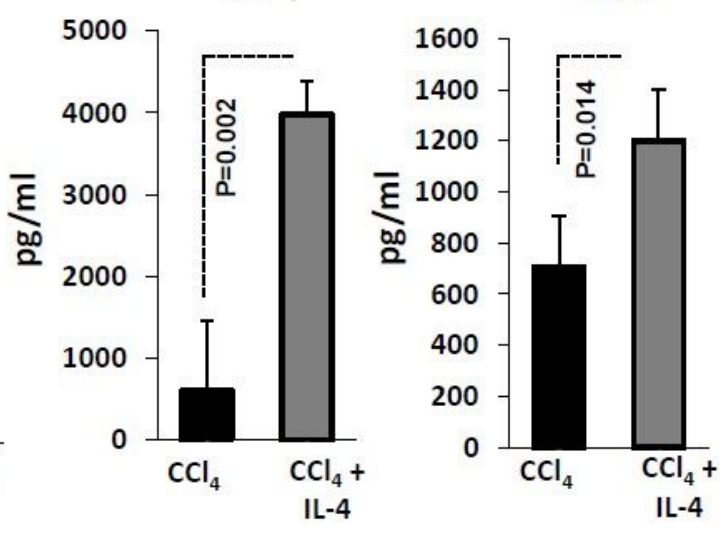

Figure 4

Interleukin-4 inhibited CCl4-induced severe fibrosis and enhanced inflammatory profile. Hepatic-fibrosis was induced in mice for 4 weeks and was compared with naïve states ( $n=5$ in each group; experiment repeated 3 times). IL-4-was induced from week 2 of CCl4 to week 4 . Representative sections of immunohistochemical liver staining with (a) H\&E, (b) Sirius red and (c) F4/80, (original magnification X100) are shown. (d) Compared to naïve mice; liver aSMA quantitation by western blot showed significant increased pattern in CCl4 mice. These elevation will inhibited with IL-4 inductions (e) Serum ALT levels were significantly increased in fibrotic mice; CCl4 with IL-4 mice showed reduced ALT levels at

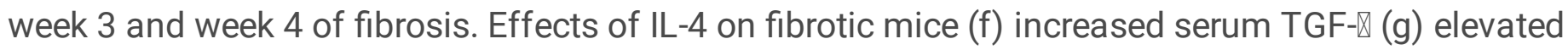
levels of INF- $\nabla$ (h) elevated levels IL-12.

\section{Supplementary Files}

This is a list of supplementary files associated with this preprint. Click to download. 
- NC3RsARRIVEGuidelinesChecklistfillable.pdf 